\title{
Locality and Universality in Gyrokinetic Turbulence
}

\author{
Bogdan Teaca, ${ }^{1, *}$ Alejandro Bañón Navarro, ${ }^{2}$ Frank Jenko, ${ }^{3}$ Stephan Brunner, ${ }^{1}$ and Laurent Villard ${ }^{1}$ \\ ${ }^{1}$ Ecole Polytechnique Fédérale de Lausanne (EPFL), Centre de Recherches en Physique des Plasmas, \\ Association Euratom-Confédération Suisse, CH-1015 Lausanne, Switzerland \\ ${ }^{2}$ Statistical and Plasma Physics, Faculty of Sciences, Université Libre de Bruxelles, \\ Campus Plaine, CP 231, B-1050 Brussels, Belgium \\ ${ }^{3}$ Max-Planck-Institut für Plasmaphysik, EURATOM Association, 85748 Garching, Germany
}

(Received 18 May 2012; published 5 December 2012)

\begin{abstract}
The nature of nonlinear interactions in gyrokinetic turbulence, driven by the ion-temperature gradient instability, is investigated using direct numerical simulations in toroidal flux tube geometry. To account for the level of separation existing between scales involved in an energetic interaction, the degree of locality of the free energy scale flux is analyzed employing Kraichnan's infrared (IR) and ultraviolet locality functions. Because of the nontrivial dissipative nature of gyrokinetic turbulence, an asymptotic level for the locality exponents, indicative of a universal dynamical regime for gyrokinetics, is not recovered and an accentuated nonlocal behavior of the IR interactions is found instead, in spite of the local energy cascade observed.
\end{abstract}

DOI: 10.1103/PhysRevLett.109.235003

PACS numbers: 52.30.Gz, 52.35.Ra, 52.65.Tt

Introduction.-The main characteristic of physical flows is given by the existence of couplings between different scales of motion $(\ell)$, described mathematically by nonlinear terms, which lead to a turbulent state for a sufficiently large interval of excited scales. While the range of scales available to the flow depends on the boundary conditions and on the nature of the flow itself, the redistribution of information among different scales is only due to the nonlinear terms. As such, the redistribution mechanism is expected to have a universal behavior for intervals of scales for which the linear terms are negligible, i.e., the inertial zone. This picture stands at the basis of the study of turbulence for hydrodynamical and electrically conductive fluids. In principle, gyrokinetic (GK) turbulence makes no exception to this picture [1,2], albeit with a series of complications due to the nature of the linear terms.

The gyrokinetic formalism is pertinent for the study of multispecies plasmas in the presence of strong magnetic guide fields [3]. By eliminating exactly the gyration phase of charged particles around the magnetic field lines [4], the dynamical space can be reduced from six dimensions to five. From the start, it is seen that the constraint imposed by the magnetic guide field on the charged flow creates an anisotropy in the system $\left(\ell=\left\{\ell_{\perp}, \ell_{\|}\right\}\right)$. The scalings in the two directions are linked as a result of causality, a hypothesis known as critical balance, recently used in the scaling of plasma turbulence $[5,6]$. The current work concentrates on the analysis of the perpendicular spatial structures $\left(\ell_{\perp}\right)$ of the fluctuations.

To understand the dynamics introduced by the nonlinear term, the scale redistribution of free energy (a GK ideal invariant, i.e., a global quantity that remains constant in time in the absence of source and sink effects) is usually investigated. Different works reported that the exchange of energy takes place between closest neighbor dyadic structures [7-9]. However, although the energy exchanges are local, the question regarding the locality of the interactions was never addressed, i.e., the fact that the local exchanges of energy might be generated by the interaction of highly separated (nonlocal) scales. In this Letter, we describe a quantitative way of asserting the degree of locality for GK turbulence. The idea of locality can be seen as the disparity of scales contributing to a nonlinear interaction. For a given energy flux through a scale, the degree to which each scale contributes to the mentioned flux represents a useful assertion of locality of interactions $[10,11]$. For the interaction to be local, the contribution of highly separated scales should be small and decrease fast with the increase in separation.

For classical fluid turbulence, the separation of the forced and dissipative scale ranges leads to the existence of a natural inertial range that possesses a unique locality exponent, which asymptotes dynamically to a $4 / 3$ value [12]. However, in the case of GK turbulence and for fusion plasma configurations in particular, each instability that generates a time unstable mode (energy source) is also accompanied, at the same scale, by an ensemble of time stable modes (energy sinks). These stable modes, known as linear damped eigenmodes, are nonlinearly coupled to the unstable modes and are responsible for an additional energy dissipation route [13]. Therefore, this dissipation mechanism acts at a scale comparable to the forcing and, as such, does not require the existence of a classical nonlinear cascade. Thus, the existence of a nonlinear cascade process is made possible only if the energy injected by the unstable modes is greater than the energy dissipated locally by the linear damped eigenmodes. Moreover, since the dissipation tends to permeate strongly the forced range 
and take over from the force as the dominant effect (characteristic time wise) for smaller scales, the nonlinear cascade process occurs inside a dissipation range [14,15]. All these effects add a novel quality and nontrivial complications to the present study, compared to classical fluid turbulence.

Gyrokinetic simulations.-In the present work, numerical solutions of the nonlinear gyrokinetic equations in flux-tube $(\hat{s}-\alpha)$ geometry [16] are analyzed using a field-aligned coordinate system $\left(x, y, z, v_{\|}, \mu\right)$ with $(256$, $128,16,48,16)$ points in each direction, respectively. In this nonorthogonal coordinate system, the $x$ label refers to the magnetic flux surface and the $y$ label identifies different field lines lying on the same flux surface, while the $\hat{\mathbf{z}} \sim \nabla x \times \nabla y$ direction coincides with that of the equilibrium magnetic guide field $(\mathbf{B})$. The solutions are obtained by the use of the GENE code [17] for ion-temperature gradient driven GK turbulence with physical parameters corresponding to the Cyclone base case (CBC) [18]. For a better understanding of the nonlinear dynamics involved, the analysis is limited to the simple scenario of electrostatic fluctuations generated by a single ion species (the species index will be omitted) and adiabatic electrons. For more technical details see Ref. [14].

Considering that the total ion distribution function $F$ is split into an appropriately normalized Maxwellian part $F_{0}$ and a perturbed part $f$, the nonadiabatic contribution of the ion distribution function is given as $h=f+\left(Z \bar{\phi}_{1} / T_{0}\right) F_{0}$, where $\bar{\phi}_{1}$ is the gyroaveraged self-consistent electrostatic potential (linear in $f$ ) found from the gyrokinetic Poisson equation, $T_{0}$ is the ion background temperature (normalized to the electron temperature), and $Z$ is the electric charge. Symbolically, the time $(t)$ evolution equation for the perturbed distribution function reads

$$
\frac{\partial f}{\partial t}=G[f]+L_{C}[f]+L_{\|}[f]+D[f]+N[f, f],
$$

where $G[f]$ is the contribution from the normalized background density $\left(n_{0}\right)$ and temperature gradients. It represents the driving mechanism for GK turbulence and it is responsible for the injection of free energy into the system. The second term, $L_{C}[f]$, appears due to magnetic curvature and gradients and the third term, $L_{\|}[f]$, contains the parallel dynamics involving magnetic trapping and linear Landau damping and pumping effects. The fourth term, $D[f]$, contains the effects due to collisions through the use of a linearized Landau collision operator for ion-ion selfcollisions [19]. The last term represents the $\mathbf{E} \times \mathbf{B}$ nonlinear term,

$$
N[f, f]=-\mathbf{v}_{E} \cdot \nabla h=\frac{\partial \bar{\phi}_{1}}{\partial y} \frac{\partial h}{\partial x}-\frac{\partial \bar{\phi}_{1}}{\partial x} \frac{\partial h}{\partial y},
$$

where $\mathbf{v}_{E}=\hat{\mathbf{z}} \times \nabla \bar{\phi}_{1}$ has been used. For the explicit forms of the terms entering Eq. (1) see Refs. [8,14].
Free energy balance.-In this formulation, the global free energy contained in the system is defined as $\mathcal{E}=\frac{1}{2} \int d x d y d \Theta \frac{T_{0}}{F_{0}} h f$ where $d \Theta=\left(\pi B_{0} n_{0}\right) d z d v_{\|} d \mu$. To analyze the excitation degree of perpendicular turbulent scales, an integral over the $d \Theta$ infinitesimal element and a Fourier decomposition of the remaining $(x, y)$ space are performed. Each scale of length $\left(\ell_{\perp}\right)$ can now be easily identified by the norm $\left(k \sim \ell_{\perp}^{-1}\right)$ of the wave vector $(\mathbf{k} \equiv$ $\mathbf{k}_{\perp}$ ) based in the $k_{x}, k_{y}$ space (units of inverse ion Larmor radius). As for any quadratic quantity, the free energy spectral density can be considered $\left(\mathcal{E}=\sum_{\mathbf{k}} \mathcal{E}^{\mathbf{k}}\right)$, for which the balance equation reads

$$
\partial_{t} \mathcal{E}^{\mathbf{k}}=G^{\mathbf{k}}+\mathcal{L}^{\mathbf{k}}+\mathcal{D}^{\mathbf{k}}+\mathcal{T}^{\mathbf{k}},
$$

where in the rhs of Eq. (3) the terms $\mathcal{A}^{\mathbf{k}} \equiv\left\{G^{\mathbf{k}}, \mathcal{L}^{\mathbf{k}}, \mathcal{D}^{\mathbf{k}}\right\}$ are found as $\mathcal{A}^{\mathbf{k}}=\int d \Theta \frac{T_{0}}{F_{0}} h^{\mathbf{k}} A^{-\mathbf{k}}$ using the spectral form of the evolution equation for the perturbed distribution function Eq. (1), with $A^{\mathbf{k}} \equiv\left\{G^{\mathbf{k}}, L^{\mathbf{k}}=L_{C}^{\mathbf{k}}+L_{\|}^{\mathbf{k}}, D^{\mathbf{k}}\right\}$. While in Eq. (3) the linear quantities $G, \mathcal{L}$, and $\mathcal{D}$ are defined involving only $\mathbf{k}$ local modes and their complex conjugates, for the term generated by the nonlinear product $\mathcal{T}^{\mathbf{k}}$ different wave number modes enter in the definition,

$$
\mathcal{T}^{\mathbf{k}}=\sum_{\mathbf{p}} \sum_{\mathbf{q}} \mathcal{T}^{\mathbf{k}, \mathbf{p}, \mathbf{q}} \delta_{\mathbf{k}+\mathbf{p}+\mathbf{q}}
$$

The Kronecker delta $\delta_{\mathbf{k}+\mathbf{p}+\mathbf{q}}$ selects only interactions that occur between a triad of modes which obey the resonance condition, $\mathbf{k}+\mathbf{p}+\mathbf{q}=\mathbf{0}$. The transfer that takes place for a single triad, known as the triad transfer, is defined as

$\mathcal{T}^{\mathbf{k}, \mathbf{p}, \mathbf{q}}=\int d \Theta \frac{T_{0}}{2 F_{0}}\left[q_{x} p_{y}-q_{y} p_{x}\right]\left[\bar{\phi}_{1}^{\mathbf{q}} h^{\mathbf{p}}-\bar{\phi}_{1}^{\mathbf{p}} h^{\mathbf{q}}\right] h^{\mathbf{k}}$,

where the symmetry in modes $q$ and $p$ is written explicitly [20]. At the triad level, the free-energy conservation by the nonlinear interaction can be written as, $\mathcal{T}^{\mathbf{k}, \mathbf{p}, \mathbf{q}}+\mathcal{T}^{\mathbf{p}, \mathbf{q}, \mathbf{k}}+\mathcal{T}^{\mathbf{q}, \mathbf{k}, \mathbf{p}}=0$

Shell transfers. - Although the triad transfers contain the complete physical information related to the energetic coupling of scales, the sheer number of transfers involved makes them impractical in any direct manner. To ease our work, we decompose the spectral space into a series of structures (which are field aligned as a result of our coordinate system choice) and analyze the transfers that occur among them. The structure boundaries $s_{K} \equiv\left(k_{K-1}, k_{K}\right]$ are typically given as a power law in terms of the wave number $k$, here $k_{K}=k_{0} \times 2^{(K-1) / \Delta}$. The filtered ion distribution function $h^{K}$ and filtered electric potential $\bar{\phi}^{K}$ are found to be

$$
\left\{h, \bar{\phi}_{1}\right\}^{K}(\mathbf{k})= \begin{cases}\left\{h, \bar{\phi}_{1}\right\}(\mathbf{k}), & k \in s_{K} \\ 0, & k \notin s_{K}\end{cases}
$$

For the CBC simulation considered, $k_{0}=0.258$ and selecting $\Delta=5$ results in $N=30$ shells. In real space, the total 
information can be recovered by summing over the inverse Fourier transform of each shell-filtered contribution.

The triple-shell transfer occurring between the shell filtered quantities can be computed as

$$
\mathcal{S}^{K, P, Q}=\sum_{\mathbf{q} \in s_{Q}} \sum_{\mathbf{p} \in s_{P}} \sum_{\mathbf{k} \in s_{K}} \mathcal{T}^{\mathbf{k}, \mathbf{p}, \mathbf{q}} \delta_{\mathbf{k}+\mathbf{p}+\mathbf{q}}
$$

and represents the basic information available to us for analysis. Knowing $\mathcal{S}^{K, P, Q}$ allows us to compute all other relevant nonlinear transfer quantities. By summing over all possible shells $Q$ we can obtain the shell-to-shell transfer $\left(\mathcal{P}^{K, P}\right.$; implicitly defined below and analyzed previously [8]) and the nonlinear transfer spectra by summing furthermore over $P$,

$$
\mathcal{T}^{K}=\sum_{P} \mathcal{P}^{K, P}=\sum_{P} \sum_{Q} \mathcal{S}^{K, P, Q} .
$$

Numerically, when summing the transfer [Eq. (8)] over $K$, which is equivalent to integrating the nonlinear transfer over the entire space, we obtain zero (comparable to machine precision).

The spectral density contributions entering in the free energy balance equation, for perpendicular characteristic scales $k_{c}$ (units of inverse ion Larmor radius) are presented in Fig. 1(a). It is interesting to note that while the spectral density $\mathcal{L}_{C}^{k}$ is found to be zero, the $\mathcal{L}_{\|}^{k}$ term, although it integrates to zero globally, contributes to the overall linear term $\left(\mathcal{L}^{k}\right)$ spectral form for time saturated states. This is important as the nonlinear transfer spectral density $\mathcal{T}^{k}$ is balanced by the sum of all the linear terms. Here, the transition from a net energy injection to a net energy dissipation regime occurs at $k_{c} \sim 1$. The subsequent nonlinear transfers between scales can be seen as taking place under the constraint of a given transfer spectra.

From Fig. 1(a), the presence of the dissipation term at all scales is obvious. The fact that a driving mechanism (occurring through the unstable eigenmodes) acts at the same scale as the dissipative effects (occurring through the stable, damped eigenmodes) signifies that the information injected into the system (mainly in the $k_{c}<1$ range) and the information cascaded down nonlinearly are different. Moreover, since both stable and unstable eigenmodes are a result of the same GK system of equations, the two effects are intertwined, which prevents us from modifying the drive without modifying the dissipation. This represents a big change compared to classical turbulence where the two terms act primarily at different wave numbers and are unlinked. Therefore, we refer to GK turbulence as having a nontrivial dissipative nature.

Locality functions.-The locality functions are defined from the triple transfers as a way to measure the nonlocality degree of the triads which contribute to the energy scale flux. The flux through a scale (here, shell boundaries $k_{c}$ ) is defined by partially summing the transfer spectra $\mathcal{T}^{K}$,

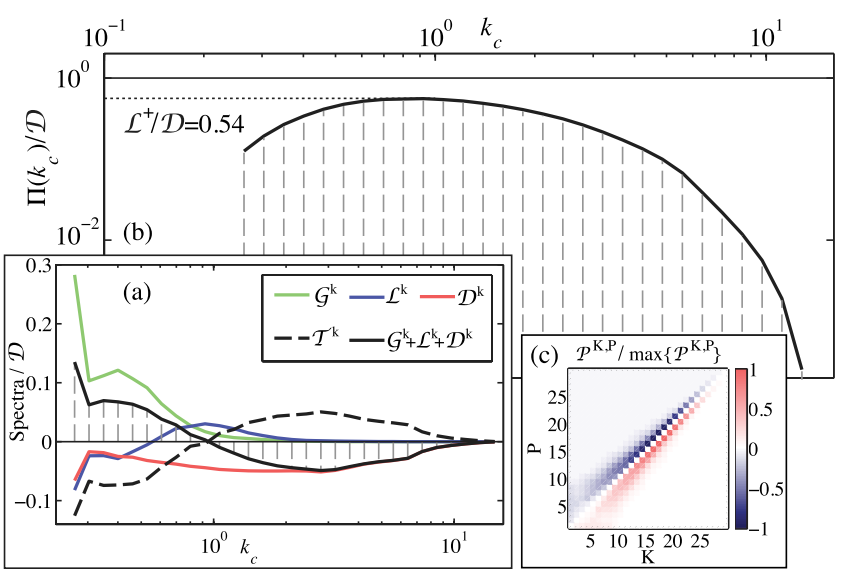

FIG. 1 (color online). The free energy rhs terms [of Eq. (3)] spectra (a) and the free energy flux across the shell boundaries (b) normalized by the total dissipation rate $\mathcal{D}$. The vertical dashed lines represent the shell boundaries. The insert picture (c) depicts the shell-to-shell transfer for this run (negative values for $P>K$ ); for details see Ref. [8].

$$
\Pi\left(k_{c}\right)=\sum_{K=c+1}^{N} \mathcal{T}^{K}=\sum_{K=c+1}^{N} \sum_{P=1}^{N} \sum_{Q=1}^{N} \mathcal{S}^{K, P, Q} .
$$

In Fig. 1(b) we show the free energy flux across the perpendicular shell wave numbers $k_{c}$. Since the source term contribution $G^{k}$ is spread over a large interval, the flux across a scale $k_{c}$ builds up slowly to its cascade saturated value (achieved at $k_{c} \sim 1$ ). Moreover, since the dissipation range is quite wide and permeates into the injection range, a true inertial range flux value cannot be identified as the plateau on the flux, Fig. 1(b). In fact, the scale flux plateau level is given by $\mathcal{L}^{+}$, representing the sum of the positive part of the linear contribution $G^{k}+\mathcal{L}^{k}+\mathcal{D}^{k}$, here the first 10 shells. The $\mathcal{L}^{+} / \mathcal{D}$ ratio clearly shows that only a fraction $(54 \%)$ of the energy injected into the system contributes to the nonlinear cascade.

From the definition given in Eq. (9), we see that the scale flux through $k_{c}$ depends on all the scales with wave numbers smaller than $k_{c}$ giving energy to all the possible scales denoted by wave numbers larger than $k_{c}$. However, is this contribution to the flux arising primarily from scales close in value to $k_{c}$ or from scales with much smaller wave number values? And, regardless of where the energy comes from in respect to $k_{c}$, is this energy going to scales of immediately larger wave numbers or scales of much larger wave numbers? These two questions represent fundamental questions for any nonlinear system and are answered through the use of locality functions. Intuitively, these functions measure a flux constructed by disregarding a given range of scales. The ratio compared to the total physical scale flux indicates the importance of the removed scales to the nonlinear interaction process.

Formally, knowing the flux, the infrared (IR) locality function is defined by taking a probe wave number boundary $k_{p}$, so that $k_{p} \leq k_{c}$, 


$$
\Pi_{\text {ir }}\left(k_{p} \mid k_{c}\right)=\sum_{K=c+1}^{N}\left[\sum_{P=1}^{N} \sum_{Q=1}^{p}+\sum_{P=1}^{p} \sum_{Q=p+1}^{N}\right] \mathcal{S}^{K, P, Q} .
$$

It measures the contribution to the flux through $k_{c}$ from triads of modes with at least one wave number less than $k_{p}$. In the second term, the sum over shell $Q$ starts from $p+1$ to avoid double counting. In the limit $k_{p} \rightarrow k_{c}$, we recover the flux across the cutoff wave number $k_{c}$. It is customary to normalize the locality functions to the flux trough $k_{c}$, in which case a value of one is obtained for $k_{p}=k_{c}$ and less than one for $k_{p} / k_{c}<1$. Although the IR functions have a clear interpretation as the ratio of energy contributed to the flux through scale $k_{c}$ coming only from larger and larger scales, it should be remembered that for $k_{p} / k_{c} \ll 1$ the transfers can only take place between the most nonlocal triads, i.e., triads with one wave vector leg much smaller compared with the other two. Therefore, these functions can provide information regarding the locality of the nonlinear interaction.

A similar definition is made for the ultraviolet (UV) locality functions, $k_{c} \leq k_{p}$,

$$
\Pi_{\mathrm{uv}}\left(k_{p} \mid k_{c}\right)=\sum_{K=1}^{c}\left[\sum_{P=1}^{N} \sum_{Q=p+1}^{N}+\sum_{P=p+1}^{N} \sum_{Q=1}^{p}\right] \mathcal{S}^{K, P, Q},
$$

which measures the contribution to the flux through $k_{c}$ from triads of modes with at least one wave number greater than $k_{p}$, therefore providing information regarding the locality makeup of a scale $k_{c}$ in relation with smaller and smaller scales.

Looking at the plot of $\Pi_{\text {ir }}\left(k_{p} \mid k_{c}\right) / \Pi\left(k_{c}\right)$ as a function of $k_{p} / k_{c}$ and $\Pi_{\mathrm{uv}}\left(k_{p} \mid k_{c}\right) / \Pi\left(k_{c}\right)$ as a function of $k_{c} / k_{p}$ will reveal information related to the locality characteristic of the nonlinear terms. The collapse of the locality functions dependence on $k_{p}$ for different values of $k_{c}$ represents a clear sign of self-similarity of the nonlinear interactions, which implies a dominance of the nonlinear terms in regard to the linear ones. Moreover, if the mentioned collapse exhibits a slope (in a log-log scale), then a state of asymptotic locality can be inferred, i.e., the nonlinear interactions saturate dynamically to the same level, no matter how large the turbulence level becomes. From our simulations (see Fig. 2) none of these two behaviors can be clearly observed.

To accentuate the scaling tendencies of the IR and UV locality functions, we perform an additional run, increasing the sub-Larmor range of scales available by taking half the computational box size in $x$ of a typical CBC run (the maximum wave number is now double). A hyperdiffusivity model [14] is used instead of a collisional operator to halve the computational costs. The number of shells is still $N=30$, however $k_{0}=0.523$. For this run, the IR and UV locality functions are presented in Fig. 3. Without a perfect collapse, the tendency of the IR exponent to reach a $1 / 12$ value can be seen better, while the UV locality

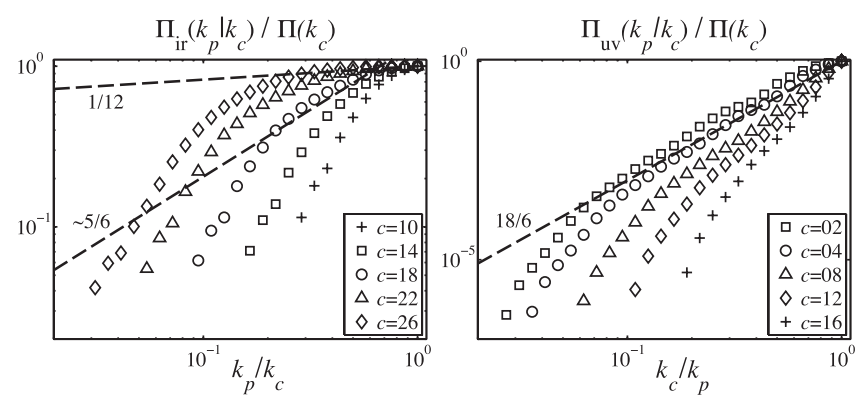

FIG. 2. The IR and UV locality functions, displayed for selected cutoff wave numbers identified by the shell index $c$. Dashed lines equal or proportional to different power laws of the abscissae are displayed for reference.

functions collapse region has a 9/6 slope in spite of the overall slope of $18 / 6$.

Scaling considerations. - Theoretically, an exponent for the IR and UV locality functions can be determined for an infinitely long inertial range. Considering the $\mathbf{v}_{E} \cdot \nabla h$ form of the nonlinearity, the triple-shell transfer is found to have the form $S^{K, P, Q} \sim\left\langle h^{K}\left(\mathbf{v}_{E}^{Q} \cdot \nabla\right) h^{P}\right\rangle$, where the angle brackets refer to volume averaging. Employing similar arguments as in Ref. [21] regarding the smoothness of scale filtered quantities, we determine a theoretical bound for the triple-shell transfer as, $S^{K, P, Q} \leq\left\langle\left|h^{K}\right|\right\rangle\left\langle\left|\mathbf{v}_{E}^{Q}\right|\right\rangle\left\langle\left|\nabla h^{P}\right|\right\rangle$. The scaling of the nonadiabatic part of the ion distribution function $\left(\left\langle\left|h^{K}\right|\right\rangle \sim k_{K}^{-1 / 6}\right)$ and the $\mathbf{E} \times \mathbf{B}$ drift velocity scaling $\left(\left\langle\left|\mathbf{v}_{E}^{K}\right|\right\rangle \sim k_{K}^{-4 / 6}\right)$ were given for two-dimensional GK turbulence by Ref. [7], from the analysis of the self-similarity statistical symmetry of their respective increments. The triple-shell transfer is bounded as $S^{K, P, Q} \leq$ (const) $k_{Q}^{-4 / 6} k_{P}^{1-1 / 6} k_{K}^{-1 / 6}$.

Assuming a local transfer among dyadic shells, i.e., shells of the form $\left[k_{K} / 2, k_{K}\right]$, the upper bound of the transfer to a scale $k_{K}$ from larger ones $\left(k_{P}<k_{K} / 2\right)$ is proportional to $\left\langle\left|h^{K}\right|\right\rangle\left\langle\left|\mathbf{v}_{E}^{[K / 2-P, K+P]}\right|\right\rangle\left\langle\left|\nabla h^{P}\right|\right\rangle$, where the wave number conservation in triad interactions has been considered. As such, the level of energetic interaction

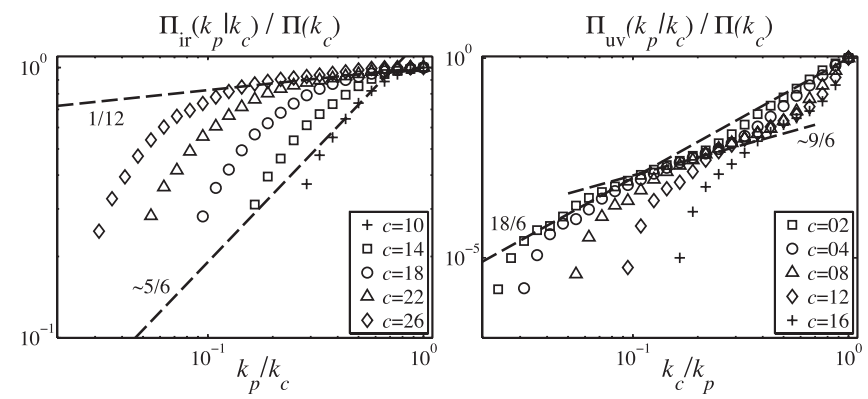

FIG. 3. The IR and UV locality functions ("half-box" run), displayed for selected cutoff wave numbers identified by the shell index $c$. 
between two scales can be seen as having the upper bound $k_{P}^{5 / 6} k_{K}^{-5 / 6}$. Similarly, the energy transfer from this scale to smaller ones $\left(k_{P}>2 k_{K}\right)$ is bounded by $k_{P}^{-5 / 6} k_{K}^{5 / 6}$. These transfer limits in turn translate as $\left(k_{p} / k_{c}\right)^{ \pm 5 / 6}$ scalings for the IR and UV locality functions.

Conclusions and discussions.-Although an asymptotic $5 / 6$ scaling of the IR and UV locality functions seems plausible and would indicate a more local interaction compared to magnetohydrodynamic turbulence $(2 / 3$ value, Refs. [11,21]) but more nonlocal compared to fluid turbulence (4/3), these values cannot be clearly identified from our simulations. First, we need to consider that the theoretical $5 / 6$ exponent is found in the limit of an infinite inertial range, an ansatz not verified in any range for GK turbulence. In spite of the local energy cascade [see Fig. 1(c)], due to dissipation, the interaction of a given scale with smaller ones will be strongly damped, increasing the scaling of the UV locality functions. The same scale will itself be damped compared to the larger scales, decreasing the IR locality exponent.

An effective nonlocal IR contribution signifies a dependence of GK turbulence on the type of instability driving it, while a stronger local UV depicts an insensitivity of gyrokinetics large scales on the small scales and therefore the type of collision mechanism employed. This last observation reinforces the practice of using numerical hyperdiffusivity terms for modeling collisional effects in GK turbulence.

The question of locality is also directly linked with the universality of GK turbulence. Although the energy cascade is local and power law energy spectra are typically reported in the literature [6-8], the strong dependence of small scales on the flow characteristics at large scales implies the need for a larger interval of scale separation between the two before they become decoupled. As such, locality reflects the strength of the decorrelation mechanism introduced by the cascade, needed to ensure the universality at small scales. For GK turbulence, the universality of small scales is apparently weakened and it may not be achieved for some practical interest problems.

B.T. is supported by the HP2C project, CSCS, Switzerland. A.B.N. is supported by Association EURATOM-Belgium state contract. Results were achieved using the computing resources of HPC-FF and JUGENE (through PRACE, including Tier-0), Jülich, Germany. The research leading to these results has received funding from the European Research Council under the European Union's Seventh Framework Programme (FP7/20072013)/ERC Grant agreement No. 277870.

*bogdan.teaca@epfl.ch

[1] A. A. Schekochihin, S. C. Cowley, W Dorland, G. W. Hammett, G. G. Howes, G. G. Plunk, E. Quataert, and T. Tatsuno, Plasma Phys. Controlled Fusion 50, 124024 (2008).

[2] T. Tatsuno, W. Dorland, A. Schekochihin, G. Plunk, M. Barnes, S. Cowley, and G. Howes, Phys. Rev. Lett. 103, 015003 (2009).

[3] J. A. Krommes, Annu. Rev. Fluid Mech. 44, 175 (2012).

[4] A. J. Brizard and T. S. Hahm, Rev. Mod. Phys. 79, 421 (2007).

[5] A. A. Schekochihin, S. C. Cowley, W. Dorland, G. W. Hammett, G. G. Howes, E. Quataert, and T. Tatsuno, Astrophys. J. Suppl. Ser. 182, 310 (2009).

[6] M. Barnes, F. Parra, and A. Schekochihin, Phys. Rev. Lett. 107, 115003 (2011).

[7] G. G. Plunk, S.C. Cowley, A. A. Schekochihin, and T. Tatsuno, J. Fluid Mech. 664, 407 (2010).

[8] A. B. Navarro, P. Morel, M. Albrecht-Marc, D. Carati, F. Merz, T. Görler, and F. Jenko, Phys. Rev. Lett. 106, 055001 (2011).

[9] G. G. Plunk and T. Tatsuno, Phys. Rev. Lett. 106, 165003 (2011).

[10] R. H. Kraichnan, J. Fluid Mech. 5, 497 (1959).

[11] B. Teaca, D. Carati, and J. A. Domaradzki, Phys. Plasmas 18, 112307 (2011).

[12] Y. Zhou, Phys. Fluids 5, 1092 (1993).

[13] D. R. Hatch, P. Terry, F. Jenko, F. Merz, and W. Nevins, Phys. Rev. Lett. 106, 115003 (2011).

[14] A. B. Navarro, P. Morel, M. Albrecht-Marc, D. Carati, F. Merz, T. Görler, and F. Jenko, Phys. Plasmas 18, 092303 (2011).

[15] P. W. Terry and V. Tangri, Phys. Plasmas 16, 082305 (2009).

[16] X. Lapillonne, S. Brunner, T. Dannert, S. Jolliet, A. Marinoni, L. Villard, T. Görler, F. Jenko, and F. Merz, Phys. Plasmas 16, 032308 (2009).

[17] F. Jenko, W. Dorland, M. Kotschenreuther, and B. N. Rogers, Phys. Plasmas 7, 1904 (2000).

[18] A. M. Dimits et al., Phys. Plasmas 7, 969 (2000).

[19] F. Merz, Ph.D. thesis, Universität Münster, 2009.

[20] M. Nakata, T.-H. Watanabe, and H. Sugama, Phys. Plasmas 19, 022303 (2012).

[21] H. Aluie and G. L. Eyink, Phys. Rev. Lett. 104, 081101 (2010). 